\title{
Comparing the Heterogeneity of Copper-Binding Characteristics for Two Different-Sized Soil Humic Acid Fractions Using Fluorescence Quenching Combined with 2D-COS
}

\author{
Jin Hur and Bo-Mi Lee \\ Department of Environment and Energy, Sejong University, Seoul 143-747, Republic of Korea
}

Received 14 July 2011; Accepted 25 August 2011

Academic Editor: Richard Brown

\begin{abstract}
Heterogeneous distributions of copper-binding characteristics were compared for two ultrafiltered size fractions of a soil HA using fluorescence quenching combined with two-dimensional correlation spectroscopy (2D-COS). The apparent shapes of the original synchronous fluorescence spectra and the extent of the fluorescence quenching upon the addition of copper were similar for the two fractions. The stability constants calculated at their highest peaks were not significantly different. However, the 2D-COS results revealed that the fluorescence quenching behaviors were strongly affected by the associated wavelengths and the fraction's size. The spectral change preferentially occurred in the wavelength order of $467 \mathrm{~nm} \rightarrow 451 \mathrm{~nm} \rightarrow 357 \mathrm{~nm}$ for the 1$10 \mathrm{~K}$ fraction and of $376 \mathrm{~nm} \rightarrow 464 \mathrm{~nm}$ for the $>100 \mathrm{~K}$ fraction. The extent of the binding affinities exactly followed the sequential orders interpreted from the 2D-COS, and they exhibited the distinctive ranges of the logarithmic values from 5.86 to 4.91 and from 6.48 to 5.95 for the $1-10 \mathrm{~K}$ and the $>100 \mathrm{~K}$ fractions, respectively. Our studies demonstrated that fluorescence quenching combined with 2D-COS could be successfully utilized to give insight into the chemical heterogeneity associated with metal-binding sites within the relatively homogeneous HA size fractions.
\end{abstract}

KEYWORDS: humic substances (HSs), two-dimensional correlation spectroscopy (2D-COS), fluorescence quenching, copper binding, Ryan-Weber model, heterogeneity 


\section{INTRODUCTION}

The accumulation and the mobility of heavy metals in soil environments are strongly affected by the presence of soil organic matter (SOM), in which humic substance (HS) is a major component [1]. The ability of HS to bind metal ions is largely attributed to oxygenated reactive groups attached to aliphatic and/or aromatic structures in HS [2]. In general, carboxylic and phenolic groups in HS operate as weak and strong binding sites for metals, respectively [3]. Previous studies have shown that carboxylic content and aromatic structures of HS were closely correlated with metal-binding capacities at a neutral $\mathrm{pH}$ condition $[4,5]$. Condensed aromatic structures in HS structures are known to favor to form highly stable complexes with metal ions by rendering a phthalic acidlike and/or a salicyclic acidlike binding sites $[1,6]$. Characterization of metal-HS interactions is viewed as an essential element for assessing the remediation of metal-contaminated soils as well as for predicting the fate of the inorganic pollutants in natural environments.

Fluorescence spectroscopy has been widely applied to capture different types of organic carbon moieties heterogeneously distributed in HS. It is highly recommended for probing various HS functionalities because it provides a wealth of information on the molecular structures and the intermolecular interactions of HS in a sensitive and a nondestructive manner. In particular, the fluorescence titration technique based on a mathematical model introduced by Ryan and Weber [7] allows one to easily determine metal stability constants and the complexing capacities. This model assumes a linear relationship between the fraction of the total ligand bound and the quenched HS fluorescence intensity and the formation of simple $1: 1 \mathrm{metal} /$ ligand complexes only. Despite some limitations of the quenching method to accurately estimate the metal binding parameters [8], it is still advantageous over other titration methods employing an ion-selective electrode in that it can afford additional spectroscopic information associated with metal binding [9].

In traditional fluorescence titration methods, metal-binding parameters have been determined using fluorescence quenching data with a single pair of excitation and emission wavelengths, which was typically selected based on the most prominent peak of the original spectrum [10,11]. However, a question is raised whether the determined binding parameters represent indeed the bulk HS consisting of heterogeneous structures and various chemical functionalities. For example, Luster et al. [12] classified the distribution of the fluorophores within a bulk HS into three types based on different fluorescence quenching behaviors upon metal addition, and they demonstrated that the calculated stability constants were dependent on the particular wavelength range. Recently, excitation-emission matrix (EEM) fluorescence spectroscopy combined with a multivariate chemometric method (e.g., parallel factor analysis) provided an insight into the presence of the heterogeneous distribution of metal-binding sites within a bulk HS [11, 13, 14].

2D-COS is a very useful tool to distinguish dissimilar spectral behaviors of a complex mixture upon the changes of external conditions [15]. It allows one to easily identify the sequence of subtle spectral changes by extending the peaks over the second dimension and the subsequent enhancement of the spectral resolution. Despite the expected benefits of using 2D-COS for complex mixtures like HS, only a few related studies have been conducted so far, in which the structural and chemical heterogeneity of dissolved organic matters (DOM) were successfully demonstrated with respect to increasing photoirradiation times [16] and to the addition of metals $[9,17]$. The synchronous fluorescence spectrum is not only easily compatible with 2D-COS but it also sufficiently presents the heterogeneous distribution of different types of fluorophores at a range of wavelengths. Therefore, 2D-COS combined with a synchronous fluorescence spectrum is expected to successfully describe the structural heterogeneity of HS with respect to a number of external factors leading to fluorescence changes.

Size fractionation is considered as a typical process to obtain relatively homogeneous HS because it is often reported that the structural, spectroscopic, and chemical differences within the bulk HS were quite pronounced among the different size fractions [18-21]. For this reason, potential chemical heterogeneity within the size fraction has long been ignored, and a few attempts have been made to compare the heterogeneous distributions of metal-binding sites for different size fractions derived from one bulk HS. It is expected that 
2D-COS combined with a synchronous fluorescence spectrum will give a more detailed characterization of metal binding behaviors with respect to the wavelengths. The objectives of this study were (1) to examine the quenching behaviors of synchronous fluorescence spectra upon the addition of copper at a range of wavelengths for two different size fractions of a soil humic acid and (2) to compare the wavelengthdependent variations of copper-binding characteristics for the two fractions employing 2D-COS. Using the physically separated HA fractions may be beneficial to explore the inherent heterogeneous distribution of copper-binding characteristics because it minimizes chemical and structural changes in HA during the preparation process [22].

\section{MATERIALS AND METHODS}

\subsection{Sample Preparation}

A soil sample was collected from Mountain Hanla in the Republic of Korea ( $\left.33^{\circ} 31^{\prime} \mathrm{N}, 126^{\circ} 32^{\prime} \mathrm{E}\right)$. The parent rock of the area is mainly trachybasalt. The clay and organic carbon contents were $64.5 \%$ and $17.9 \%$, respectively [23]. The sample represents a composite of 10 individual soils taken from the surface horizon $(0-5 \mathrm{~cm})$, which were collected within 10000 square meters from an uncontaminated and long-undisturbed area. The soil was passed through a $2 \mathrm{~mm}$ sieve to remove gravel, roots, and leaves before it was air-dried.

The procedures for the extraction and the purification of HA were well described in our prior study [21]. Two different size fractions $(1-10 \mathrm{~K}$ and $>100 \mathrm{~K})$ of the HA were obtained using an Amicon batch stirred cell of $250 \mathrm{~mL}$ capacity and Amicon ultrafiltration (UF) membranes with the molecular weight cutoffs of $1 \mathrm{~K}, 10 \mathrm{~K}$, and $100 \mathrm{KDa}$. The details of the procedure were provided in Hur and Kim [24]. The original DOC concentrations of the two prepared fractions were $91.1 \mathrm{mg} \mathrm{C} / \mathrm{L}$ and $900.8 \mathrm{mg} \mathrm{C} / \mathrm{L}$ for the $1-10 \mathrm{~K}$ and the $>100 \mathrm{~K}$ fractions, respectively.

\subsection{Dissolved Organic Carbon (DOC) and UV Absorbance Measurements}

DOC concentrations of the HA samples were measured based on a Shimadzu V-CPH analyzer. UV absorbance measurements were made using a UV-visible spectrophotometer (Evolution 60, Thermo Scientific) and a $1 \mathrm{~cm}$ quartz cuvette to determine the specific UV absorbance (SUVA) values of the samples. SUVA is equivalent to the 100-fold ratio of the UV absorbance at $254 \mathrm{~nm}$ to the DOC concentration.

\subsection{Synchronous Fluorescence Spectra}

Synchronous fluorescence spectra of the HA size fractions were measured using a luminescence spectrometer (Perkin-Elmer LS-50B). Excitation and emission slits were both adjusted to $10 \mathrm{~nm}$, and the excitation wavelengths were scanned from 250 to $600 \mathrm{~nm}$ with a constant offset $(\Delta \lambda=30 \mathrm{~nm})$. The samples were diluted to a concentration of $10.0 \mathrm{mg}$ C/L for the fluorescence measurements. A $290 \mathrm{~nm}$ cutoff filter was used to minimize second-order Raleigh scattering. The fluorescence response to a blank solution was subtracted from the spectrum of the sample. In this study, three fluorescence regions were assigned to protein-like (PLF), humic-like (HLF), and terrestrial humic-like fluorescence (THLF), each of which corresponds to the integrated areas of the fluorescence intensities at the wavelength of $250-300 \mathrm{~nm}, 300-420 \mathrm{~nm}$, and 420 $600 \mathrm{~nm}$, respectively [21]. Each relative fluorescence region over the whole fluorescence area was reported as \% PLF, \%HLF, and \% THLF, respectively. 


\subsection{D-COS Principle and Application}

2D-COS generates two types of the spectra called synchronous 2D and asynchronous 2D spectra, each of which is calculated based on the correlations between two different dynamic spectra and the cross-correlations between the dynamic spectrum and its orthogonal spectrum. The mathematical expressions of the two spectra are given as follows [24]:

$$
\begin{aligned}
& \Phi\left(v_{1}, v_{2}\right)=\frac{1}{T_{\max }-T_{\min }} \int_{T}^{T_{\max }} \tilde{y}\left(v_{1}, t\right) \times \tilde{y}\left(v_{2}, t\right) d t, \\
& \Psi\left(v_{1}, v_{2}\right)=\frac{1}{T_{\max }-T_{\min }} \int_{T_{\min }}^{T_{\max }} \tilde{y}\left(v_{1}, t\right) \times \widetilde{z}\left(v_{2}, t\right) d t,
\end{aligned}
$$

where $\Phi$ and $\Psi$ are synchronous 2D and asynchronous 2D spectra, respectively. The parameters of $v$ and $t$ represent a spectral variable and an external perturbation, respectively. $\widetilde{y}(v, t)$ is the dynamic spectrum, and $\widetilde{z}(v, t)$ is the Hilbert-transformed orthogonal spectrum.

In a contour plot of the synchronous $2 \mathrm{D}$ spectrum, the intensity of peaks located at diagonal position (i.e., autopeaks) represents the overall extent of the variation in the spectral intensity observed at $v$ during the interval between $T_{\min }$ and $T_{\max }$. Cross-peaks, which are located off the diagonal line of a synchronous 2D spectrum, represent simultaneous changes of the spectral intensities observed at two different spectral variables of $v_{1}$ and $v_{2}$. The sign of the synchronous cross-peaks indicates the direction of the spectral changes. For example, a positive cross-peak indicates the changes of the spectral intensities at two different variables proceed in the same direction whereas a negative cross-peak reveals the changes in the opposite direction. Meanwhile, the sign of an asynchronous cross-peak provides the information on the sequence of events observed with the external perturbation. If the signs of synchronous and asynchronous cross-peaks are the same, the spectral change at $v_{1}$ precedes the change at $v_{2}$. If they are different, the change at $v_{1}$ is followed by the change at $v_{2}$ (i.e., Noda's rules) $[25,26]$.

In this study, the synchronous and the asynchronous $2 \mathrm{D}$ spectra were calculated by using the algorithm based on the numerical method developed by Noda et al. [25], and the corresponding maps were generated using the same software as described elsewhere [15].

\subsection{Determination of Copper Complexing Capacities and Stability Constants}

Copper stock solutions with different concentrations $(0.2,1.0$, and $5.0 \mathrm{mM})$ were prepared using $\mathrm{Cu}\left(\mathrm{NO}_{3}\right)_{2}$. $\mathrm{HA}$ samples $(10 \mathrm{mg} \mathrm{C} / \mathrm{L})$ were transferred into polypropylene vials $(2 \mathrm{~mL})$ containing $\mathrm{pH}$-adjusted $\mathrm{KNO}_{3}$ solution $(\mathrm{pH}=6.0)$. Aliquots of the stock solutions were then spiked into a series of the vials such that the vials with varying copper concentrations from 0 to $125 \mu \mathrm{M}$ could be prepared in triplicate. The vials were placed on a shaker at low speed and allowed to equilibrate for 15 minutes. The equilibrium time was determined based on previous pieces of the literature $[2,9,11]$, in which no further fluorescence quenching was observed after the time. The synchronous fluorescence spectra of our samples were measured after the solution $\mathrm{pH}$ was readjusted to 6.0 using dilute $\mathrm{HNO}_{3}$ or $\mathrm{NaOH}$.

In this study, the Ryan-Weber nonlinear model was applied to determine the parameters related to copper binding [2]. The Ryan-Weber model equation is given by

$$
F=F_{0}+\left(F_{\mathrm{ML}}-F_{0}\right)\left(\frac{1}{2 K_{\mathrm{M}} C_{\mathrm{L}}}\right)\left[1+K_{\mathrm{M}} C_{\mathrm{L}}+K_{\mathrm{M}} C_{\mathrm{M}}-\sqrt{\left(1+K_{\mathrm{M}} C_{\mathrm{L}}+K_{\mathrm{M}} C_{\mathrm{M}}\right)^{2}-4 K_{\mathrm{M}}^{2} C_{\mathrm{L}} C_{\mathrm{M}}}\right],
$$


where $F, F_{0}$, and $F_{\mathrm{ML}}$ are the measured fluorescence intensities at $C_{\mathrm{M}}$ concentration and without metal addition and the limiting fluorescence intensity below which the intensity does not change upon the metal addition, respectively. The parameter, $C_{\mathrm{L}}$, is the stoichiometric concentration of the ligand. $K_{\mathrm{M}}$ and $C_{\mathrm{M}}$ are the conditional stability constant and the total metal concentration, respectively. Model fitting was performed using ORIGIN software (Version 6.0, Microcal Software, Inc.) to obtain the optimum values of $K_{\mathrm{M}}, C_{\mathrm{L}}$, and $F_{\mathrm{ML}}$. The complexing capacity $(\mathrm{mmol} / \mathrm{g} \mathrm{C})$ was calculated by dividing the $C_{\mathrm{L}}$ value by the DOC concentrations of the samples.

The fluorescence intensities used for the model fitting were based on the peak wavelengths of the original synchronous fluorescence spectra or the wavelength pairs of the asynchronous 2D spectra exhibiting pronounced peaks.

\section{RESULTS AND DISCUSSION}

\subsection{UV-Visible and Fluorescence Characteristics of the Two Size Fractions}

The $1-10 \mathrm{~K}$ fraction of the soil HA exhibited a slightly higher SUVA value than the $>100 \mathrm{~K}$ fraction (Table 1), suggesting the smaller size fraction contains more aromatic carbon content at the same DOC concentration although the difference was not so pronounced compared to those reported in other studies using different size fractions of terrestrial HS $[18,26]$. In this study, a much higher $E_{4} / E_{6}$ value, the absorbance ratio at $465 \mathrm{~nm}$ and $665 \mathrm{~nm}$, was observed for the size fraction of $1-10 \mathrm{~K}$ versus $>100 \mathrm{~K}$ (Table 1). The result agreed well with the molecular size of the HA fractions because $E_{4} / E_{6}$ is known to be negatively correlated with molecular weight of HS.

Synchronous fluorescence spectra of the two size fractions are shown in Figure 1. Apparently, the spectral shapes of the two fractions were very similar to each other, in which three small peaks and one major peak could be identified from the HLF and the THLF regions, respectively. The slight PLF intensities were observed for the $1-10 \mathrm{~K}$ fraction only. Other studies indicate that PLF characteristics are not likely to be prominent for soil environments where microbial transformation of terrigenous organic matters is dominant although the feature could be associated with the presence of protein and/or amino acid bound HA and/or tannin-like substances [21].

A notable difference between the two original spectra was much higher fluorescence intensities per organic carbon for the fraction of $1-10 \mathrm{~K}$ versus $>100 \mathrm{~K}$ (Figure 1). For example, the THLF peak intensity of the $1-10 \mathrm{~K}$ fraction was as much as 20 times higher than that of the $>100 \mathrm{~K}$ fraction at the same DOC concentration. Other reports also showed evidence of the heterogeneous distribution of the fluorophores among different size fractions $[27,28]$. The more intense emitting intensity of the $1-10 \mathrm{~K}$ fraction versus the $>100 \mathrm{~K}$ fractions may be attributed to relatively high content of fluorescent constituents contained in the former and/or to the potential existence of deactivation processes in the latter [27, 29].

\subsection{Changes in the Synchronous Fluorescence Spectra upon the Addition of Copper}

Changes in the synchronous fluorescence spectra upon the addition of copper were compared for the two size fractions (Figure 1). The fluorescence intensities were quenched at all the wavelengths investigated, indicating that that electronic structural changes extensively occurred in the HA fractions by forming complexes with copper [2]. The apparent quenching behaviors were similar for the two fractions. For example, for both fractions, the extent of the quenching appears to be the most pronounced at the THLF peak, and it tends to be diminished with shorter wavelengths. The THLF peaks of the two fractions both nearly disappeared after the copper was sufficiently added until no fluorescence change occurred (i.e., copper-saturated condition for the fluorophores). However, a closer examination revealed the presence of subtle differences between the spectral variations with wavelengths for the two fractions. For example, 
TABLE 1: Specific UV absorbance (SUVA) values and fluorescence distributions for the two different size fractions of a soil humic acid and the mixture.

\begin{tabular}{lccccc}
\hline Sample & SUVA $\left(\mathrm{L}(\mathrm{mg} \mathrm{C})^{-1} \mathrm{~m}^{-1}\right)$ & $E_{4} / E_{6}$ & \%PLF & \%HLF & \% THLF \\
\hline $1-10 \mathrm{~K}$ & $4.06 \pm 0.02^{1}$ & $12.4 \pm 1.9^{1}$ & $0.8 \pm 0.1$ & $38.5 \pm 0.2$ & $60.7 \pm 0.4$ \\
$>100 \mathrm{~K}$ & $3.87 \pm 0.05$ & $4.4 \pm 0.2$ & $0.0 \pm 0.0$ & $39.1 \pm 0.3$ & $60.9 \pm 0.5$ \\
\hline
\end{tabular}

${ }^{1}$ Standard deviation based on triplicate samples.

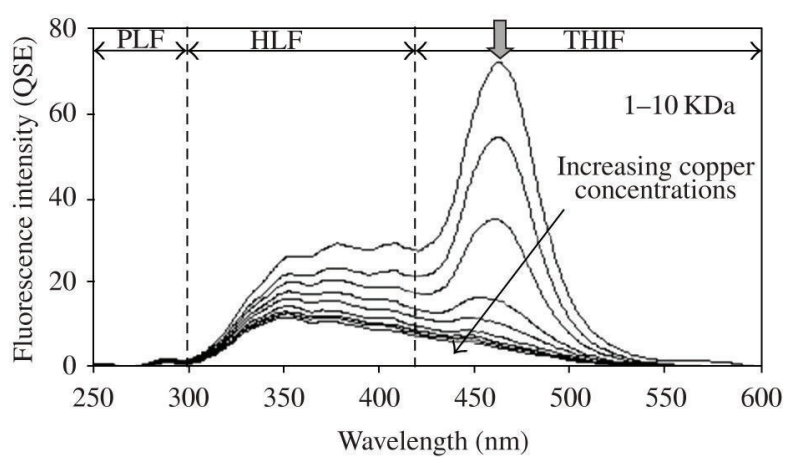

(a)



(b)



(c)

FIGURE 1: Changes in the synchronous fluorescence spectra for (a) the 1-10 KDa fraction and (b) the $>100 \mathrm{KDa}$ fraction of the soil HA upon the addition of copper. The thin long arrows refer to the direction of the increasing copper concentrations. The vertical shaded arrows indicate the locations of the wavelengths for a representative peak of each fraction.

the fluorescence intensities of the $1-10 \mathrm{~K}$ fraction tend to steadily decrease at all the wavelength ranges whereas, for the $>100 \mathrm{~K}$ fraction, the quenching behaviors were evidently dependent on the fluorescence regions. The fluorescence of the HLF region sharply decreased to a certain level of the copper addition, and little change occurred afterwards. In contrast, a steady decrease of the fluorescence intensity was observed at the THLF region (Figure 1(b) ). Our observation implies that the copper-binding sites may be unevenly distributed even within the relatively homogeneous HA size fraction, which was consistent with the findings with hydrophobic/hydrophilic fractions of DOM in our earlier report [9].

\subsection{D Correlation Fluorescence Spectroscopy}

One positive autopeak was commonly observed for the synchronous 2D spectra of the two size fractions, indicating that the spectral changes took place in the same direction at the corresponding wavelength ranges (i.e., decreasing fluorescence intensity) (Figure 2). A much higher intensity of the autopeak for the fraction 


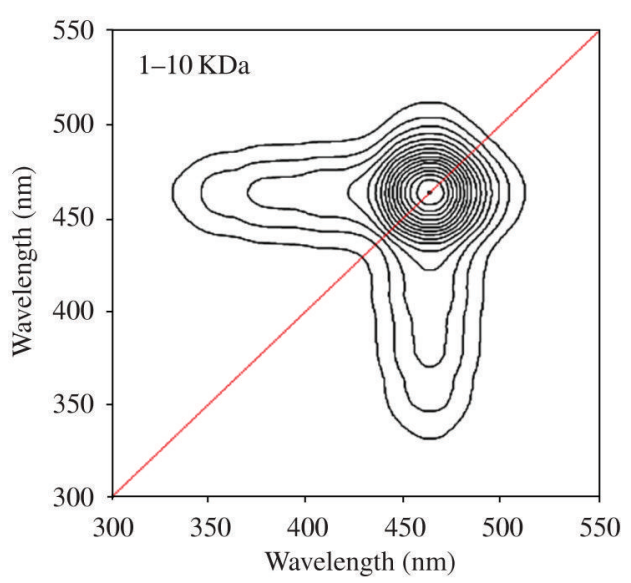

(a)

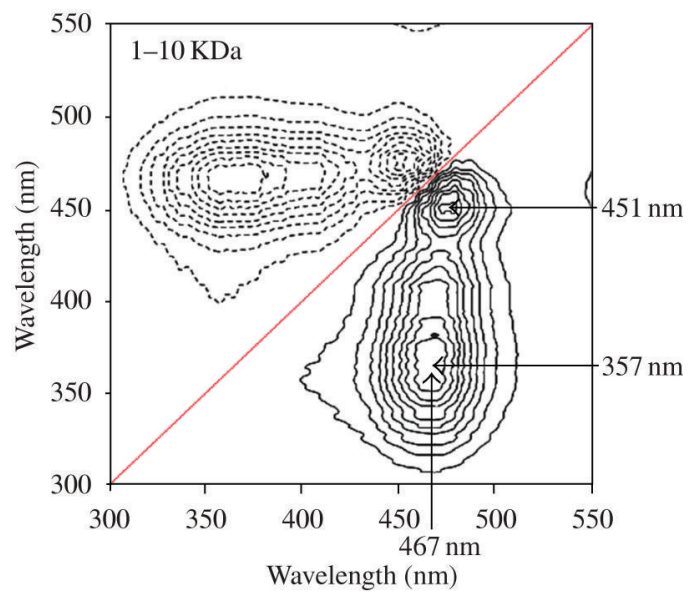

(c)

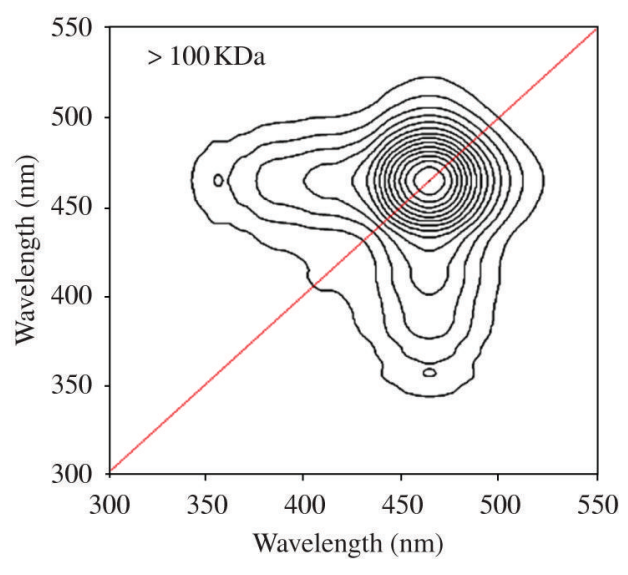

(b)

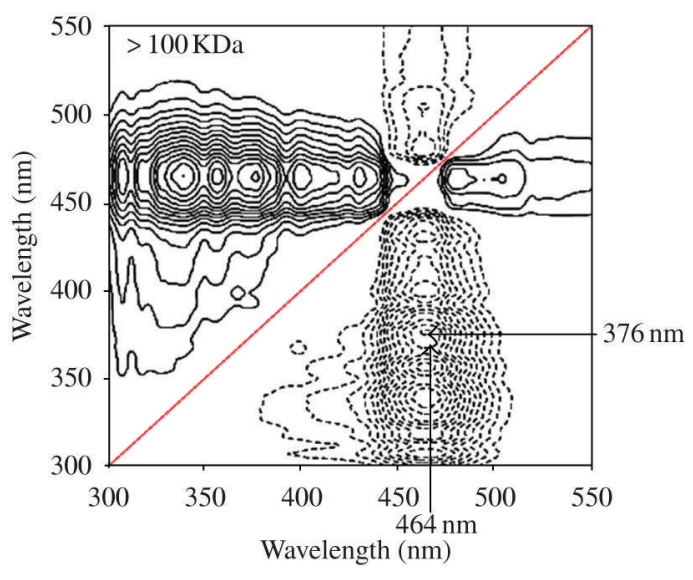

(d)

FIGURE 2: Synchronous 2D spectrum for (a) the $1-10 \mathrm{~K}$ fraction and (b) the $>100 \mathrm{~K}$ fraction. Asynchronous $2 \mathrm{D}$ spectrum for $(\mathrm{c})$ the $1-10 \mathrm{~K}$ fraction and (d) the $>100 \mathrm{~K}$ fraction of the soil HA. The arrows indicate the main peaks of the spectra, based on which the copper-binding parameters were calculated in Table 2 . The $x$ - or $y$-axis represents the excitation wavelength of the synchronous fluorescence spectra. The solid and the dashed lines represent the positive and negative signs, respectively.

of $1-10 \mathrm{~K}$ versus $>100 \mathrm{~K}$ suggests that the smaller size fraction may be more susceptible to the fluorescence quenching upon the addition of copper.

The asynchronous 2D spectra illustrated the sequence of the fluorescence changes at two different wavelengths (i.e., the changes at one wavelength lead or lag those at the other wavelength) (Figures 2(c) and 2(d) ). For the 1-10 K fraction, two positive peak bands were observed below, off the diagonal line of the asynchronous 2D spectra (Figure 2(c)). The large positive band was horizontally extended from the $x_{1}$ wavelengths of $425 \mathrm{~nm}$ to $500 \mathrm{~nm}$ and, vertically, from the $x_{2}$ wavelengths of $310 \mathrm{~nm}$ to $425 \mathrm{~nm}$, with the highest peak shown at the $x_{1} / x_{2}$ wavelengths of $467 \mathrm{~nm} / 357 \mathrm{~nm}$. According to Noda's rules [25], the location of this peak area can be interpreted as the earlier occurrence of the fluorescence quenching at the longer wavelength range (i.e., $425 \mathrm{~nm}$ to $500 \mathrm{~nm}$ ) rather than at the shorter wavelengths from $310 \mathrm{~nm}$ to $425 \mathrm{~nm}$. This finding is consistent with our previous observation of the decreased extent of the fluorescence quenching upon the copper addition with shorter wavelengths (Figure 1). The smaller positive area was centered at the $x_{1} / x_{2}$ wavelengths of $451 \mathrm{~nm} / 467 \mathrm{~nm}$, indicating that the fluorescence quenching took place at $451 \mathrm{~nm}$ prior to that at $467 \mathrm{~nm}$ (i.e., stronger binding at $451 \mathrm{~nm}$ versus $467 \mathrm{~nm}$ ). It is important to note that 
it is very difficult to recognize such a subtle difference in the spectral behaviors from the spectral changes of the original spectra. Our 2D-COS results evidenced that there might be a heterogeneous distribution of the binding sites within the size HA fraction. Based on Noda's rules, the locations of the two peak bands revealed that the fluorescence quenching occurred in the sequential order of the wavelengths of $467 \mathrm{~nm}$ $\rightarrow 451 \mathrm{~nm} \rightarrow 357 \mathrm{~nm}$. It is expected that the kinetic rates of the fluorescence quenching (i.e., stability constants) will decrease in accordance with the wavelength order.

From the asynchronous 2D spectrum of the $>100 \mathrm{~K}$ fraction, one slight positive peak and two negative large peaks were observed (Figure 2(d) ). The positive peaks were centered at $502 \mathrm{~nm} / 464 \mathrm{~nm}$, and the negative main peaks were located at $464 \mathrm{~nm} / 356 \mathrm{~nm}$ and $464 \mathrm{~nm} / 376 \mathrm{~nm}$. Based on Noda's rules, the observed spectral features can be depicted as the preferential occurrence of the fluorescence quenching in the wavelength order of $356 \mathrm{~nm} \sim 376 \mathrm{~nm} \rightarrow 502 \mathrm{~nm} \rightarrow 464 \mathrm{~nm}$. However, the sequential fluorescence behaviors associated with the wavelengths of $356 \mathrm{~nm}$ and $502 \mathrm{~nm}$ may not be obvious because the corresponding areas of the synchronous $2 \mathrm{D}$ spectrum are barely positive and/or the peaks are too small. Therefore, the sequential wavelength order of $376 \mathrm{~nm}>464 \mathrm{~nm}$ only is likely to be pronounced.

Despite the similarity in the synchronous 2D spectra of the two fractions, contrasting patterns were found for the corresponding asynchronous $2 \mathrm{D}$ spectra. For example, the fluorescence quenching at the THLF region (i.e., longer wavelength range) appears to occur earlier than that at the HLF region for the small size fraction whereas the opposite trend was observed for the large size fraction. Our combined results demonstrated that copper-binding characteristics were highly dependent on the associated wavelengths and also that the trend of the variation with wavelengths might be different depending on the fraction's size of the HA.

\subsection{Copper(II)-Binding Parameters of Two Size Fractions}

In order to quantitatively validate the wavelength-dependent distribution of the copper-binding characteristics interpreted from the 2D-COS results, copper stability constants were calculated by the best fit of the Ryan-Weber model at the wavelengths selected based on the peak locations of the asynchronous 2D spectra (Table 2 and Figure 3). For comparison, the same parameters were calculated as well based on the representative peak wavelengths of the original synchronous fluorescence spectra (Table 2).

All the titration data were fitted well with the Ryan-Weber model as indicated by the high correlation coefficients $(r>0.950)$ and the statistical significances $(P<0.001)$. Our calculated stability constants expressed by $\log K_{\mathrm{M}}$ values ranged from 4.91 to 6.48 for the two fractions. The range was comparable to those reported from other studies using terrestrial DOM [2, 12, 30, 31]. In this study, two distinctive ranges of the $\log K_{\mathrm{M}}$ values were observed for the two size fractions. The $1-10 \mathrm{~K}$ fraction showed a range of $\log K_{\mathrm{M}}$ values from 4.91 to 5.86 while the higher range from 5.95 to 6.48 was observed for the $>100 \mathrm{~K}$ fraction. The higher copper stability constant for the large size HA fraction is consistent with the result of a prior report [32]. However, the opposite trend is also reported in other studies [19, 33]. The chemical and structural difference between the size fractions relating to the stability constant needs to be further investigated.

The extent of the stability constants calculated was surprisingly consistent with the sequential orders determined from the $2 \mathrm{D}-\mathrm{COS}$ results. For the $1-10 \mathrm{~K}$ fraction, the stability constants exhibited a decreasing trend in the wavelength order of $467 \mathrm{~nm}>451 \mathrm{~nm}>357 \mathrm{~nm}$. For the $>100 \mathrm{~K}$ fraction, a decreasing trend of the $\log K_{\mathrm{M}}$ values was observed in the wavelength order of $376 \mathrm{~nm}>464 \mathrm{~nm}$. Both cases agreed well with the interpretations of the previous 2D-COS results.

It should be noted that a simple comparison of the stability constants determined at single wavelength pairs of the original synchronous fluorescence spectra may fail to understand the heterogeneous distribution of the copper-binding characteristics in HA. For example, the two stability constants calculated based on the representative wavelengths were not significantly different (i.e., $5.86 \pm 0.07$ and $5.96 \pm 0.08$ for the $1-10 \mathrm{~K}$ and the $>100 \mathrm{~K}$ fractions, resp.) in Table 2. In contrast, the 2D-COS results revealed the distinctive ranges in the values for the two fractions. 
TABLE 2: Copper-binding parameters ${ }^{1}$ calculated by the Ryan-Weber model.

\begin{tabular}{lccccc}
\hline HA size fractions & Peak wavelength $(\mathrm{nm})$ & $r$ & $F_{\mathrm{ML}}(\%)$ & $\log K_{\mathrm{M}}$ & $C C_{\mathrm{M}}\left(\mathrm{mmol}(\mathrm{gC})^{-1}\right)$ \\
\hline \multirow{3}{*}{$1-10 \mathrm{~K}$} & $357^{2}$ & $0.996^{*}$ & $40.0 \pm 2.4$ & $4.91 \pm 0.06$ & $0.63 \pm 0.29$ \\
& $451^{2}$ & $1.000^{*}$ & $7.2 \pm 6.3$ & $5.70 \pm 0.03$ & $1.33 \pm 0.14$ \\
& $462^{3}$ & $0.999^{*}$ & $6.3 \pm 0.7$ & $5.86 \pm 0.07$ & $1.17 \pm 0.09$ \\
$>100 \mathrm{~K}$ & $467^{2}$ & $0.999^{*}$ & $5.6 \pm 0.5$ & $5.86 \pm 0.05$ & $1.56 \pm 0.29$ \\
& $376^{2}$ & $0.953^{*}$ & $59.7 \pm 1.5$ & $6.48 \pm 0.52$ & $0.67 \pm 1.22$ \\
& $460^{3}$ & $0.999^{*}$ & $15.7 \pm 0.9$ & $5.96 \pm 0.08$ & $1.12 \pm 0.32$ \\
\hline
\end{tabular}

${ }^{1}$ Correlation coefficients of predicted versus observed fluorescence intensity $(r)$, relative percentage of fluorescence intensity for copper-saturated complexes $\left(F_{\mathrm{ML}}(\%)\right)$, conditional stability constants $\left(\log K_{\mathrm{M}}\right)$, and complexing capacities $\left(C C_{\mathrm{M}}\right)$ and fraction of the initial fluorescence corresponding to binding sites $(f)$.

${ }^{2}$ The peak wavelengths were selected based on peaks observed in the synchronous and asynchronous maps of 2D-COS in Figure 2.

${ }^{3}$ The peak wavelengths were selected based on the apparent shapes of the synchronous fluorescence spectra in Figure 1.

${ }^{*} P$ value $<0.001$.



(a)

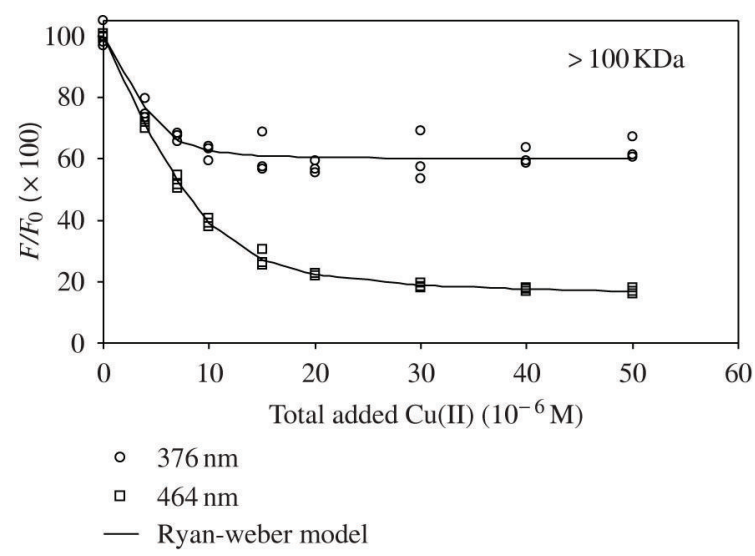

(b)

FIGURE 3: Representative fluorescence quenching curves for the (a) $1-10 \mathrm{~K}$ fraction and (b) the $>100 \mathrm{~K}$ fraction of the soil HA. The lines represent model-predicted values.

In addition to the stability constants, considerable ranges of the other binding parameters such as $F_{\mathrm{ML}}$ and $C C_{\mathrm{M}}$ values were observed within the size fraction. For example, the percentages of fluorophores that participate in the complexation reaction (i.e., $100-F_{\mathrm{ML}}$ ) ranged from $60 \%$ to $94 \%$ for the $1-10 \mathrm{~K}$ fraction and from $40 \%$ to $85 \%$ for the $>100 \mathrm{~K}$ fraction. The percentages tend to increase with longer wavelengths for both fractions. A large variation was also observed for the complexation capacities of each size fraction but no consistent trend was found with wavelengths. Unlike the stability constants, no distinction in the ranges of the binding capacities was observed between the two size fractions, which corresponded to 0.63 to $1.56 \mathrm{mmol} / \mathrm{g} \mathrm{C}$ and 0.67 to $1.12 \mathrm{mmol} / \mathrm{g} \mathrm{C}$ for the $1-10 \mathrm{~K}$ and the $>100 \mathrm{~K}$ fractions, respectively (Table 2).

\section{CONCLUSIONS}

At prominent peaks of synchronous fluorescence spectra, the extent of the fluorescence quenching upon the addition of copper was apparently similar for the two size fractions. The corresponding stability constants were not significantly different, exhibiting the logarithmic values of $5.86 \pm 0.07$ and $5.96 \pm 0.08$ for the $1-10 \mathrm{~K}$ and the $>100 \mathrm{~K}$ fractions, respectively. However, fluorescence quenching combined with 2D-COS 
revealed in detail the wavelength-dependent fluorescence quenching behaviors, in which the sequences of the fluorescence quenching were observed with the wavelength order of $467 \mathrm{~nm} \rightarrow 451 \mathrm{~nm} \rightarrow 357 \mathrm{~nm}$ for the $1-10 \mathrm{~K}$ fraction and of $376 \mathrm{~nm} \rightarrow 464 \mathrm{~nm}$ for the $>100 \mathrm{~K}$ fraction. The extent of the logarithmic values of the calculated stability constants agreed well with the sequential orders interpreted from the 2D-COS. In addition, the distinctive ranges of the logarithmic values were found, which corresponded to from 5.86 to 4.91 and from 6.48 to 5.96 for the $1-10 \mathrm{~K}$ and the $>100 \mathrm{~K}$ fractions, respectively. The higher range in the stability constants was possibly explained by more condensed aromatic structures of the larger size fraction. Our studies demonstrated that 2D-COS could be successfully utilized to obtain detailed information on the heterogeneous distribution of copper-binding characteristics within the relatively homogeneous HA size fraction, which is not easily recognized by simple comparison of the spectral quenching behaviors of the original spectra.

\section{ACKNOWLEDGMENT}

This work was supported by the National Research Foundation of Korea Grant funded by the Korean Government (MEST) (NRF-2010-0021086).

\section{REFERENCES}

[1] F. J. Stevenson, Humus Chemistry: Genesis, Composition, Reactions, John Wiley \& Sons, New York, NY, USA, 1994.

[2] C. Plaza, G. Brunetti, N. Senesi, and A. Polo, "Molecular and quantitative analysis of metal ion binding to humic acids from sewage sludge and sludge-amended soils by fluorescence spectroscopy," Environmental Science and Technology, vol. 40, no. 3, pp. 917-923, 2006.

[3] J.-P. Croué, M. F. Benedetti, D. Violleau, and J. A. Leenheer, "Characterization and copper binding of humic and nonhumic organic matter isolated from the South Platte River: evidence for the presence of nitrogenous binding site," Environmental Science and Technology, vol. 37, no. 2, pp. 328-336, 2003.

[4] K. A. Merritt and M. S. Erich, "Influence of organic matter decomposition on soluble carbon and its copperbinding capacity," Journal of Environmental Quality, vol. 32, no. 6, pp. 2122-2131, 2003.

[5] F. Amery, F. Degryse, W. Degeling, E. Smolders, and R. Merckx, "The copper-mobilizing-potential of dissolved organic matter in soils varies 10-fold depending on soil incubation and extraction procedures," Environmental Science and Technology, vol. 41, no. 7, pp. 2277-2281, 2007.

[6] K. M. Elkins and D. J. Nelson, "Spectroscopic approaches to the study of the interaction of aluminum with humic substances," Coordination Chemistry Reviews, vol. 228, no. 2, pp. 205-225, 2002.

[7] D. K. Ryan and J. H. Weber, "Fluorescence quenching titration for determination of complexing capacities and stability constants of fulvic acid," Analytical Chemistry, vol. 54, no. 6, pp. 986-990, 1982.

[8] M. D. Hays, D. K. Ryan, and S. Pennell, "A modified multisite stern-volmer equation for the determination of conditional stability constants and ligand concentrations of soil fulvic acid with metal ions," Analytical Chemistry, vol. 76, no. 3, pp. 848-854, 2004.

[9] J. Hur and B.-M. Lee, "Characterization of binding site heterogeneity of copper within dissolved organic matter fractions using two-dimensional correlation spectroscopy," Chemosphere, vol. 83, pp. 1603-1611, 2011.

[10] K. K. de Zarruk, G. Scholer, and Y. Dudal, "Fluorescence fingerprints and $\mathrm{Cu}^{2+}$-complexing ability of individual molecular size fractions in soil- and waste-borne DOM," Chemosphere, vol. 69, no. 4, pp. 540-548, 2007.

[11] T. Ohno, A. Amirbahman, and R. Bro, "Parallel factor analysis of excitation-emission matix fluorescence spectra of water soluble soil organic matter as basis for the determination of conditional metal binding parameters," Environmental Science \& Technology, vol. 42, pp. 186-192, 2008.

[12] J. Luster, T. Lloyd, G. Sposito, and I. V. Fry, "Multi-wavelength molecular fluorescence spectrometry for quantitative characterization of copper(II) and aluminum(III) complexation by dissolved organic matter," Environmental Science and Technology, vol. 30, no. 5, pp. 1565-1574, 1996. 
[13] Y. Yamashita and R. Jaffé, "Characterizing the interactions between trace metals and dissolved organic matter using excitation-emission matrix and parallel factor analysis," Environmental Science and Technology, vol. 42, no. 19, pp. 7374-7379, 2008.

[14] J. Wu, H. Zhang, P.-J. He, and L.-M. Shao, "Insight into the heavy metal binding potential of dissolved organic matter in MSW leachate using EEM quenching combined with PARAFAC analysis," Water Research, vol. 45, pp. 1711-1719, 2011.

[15] Y. M. Jung and I. Noda, "New approaches to generalized two-dimensional correlation spectroscopy and its applications," Applied Spectroscopy Reviews, vol. 41, no. 5, pp. 515-547, 2006.

[16] J. Hur, K.-Y. Jung, and Y. M. Jung, "Characterization of spectral responses of humic substances upon UV irradiation using two-dimensional correlation spectroscopy," Water Research, vol. 45, no. 9, pp. 2965-2974, 2011.

[17] K. Nakashima, S. Xing, Y. Gong, and T. Miyajima, "Characterization of humic acids by two-dimensional correlation fluorescence spectroscopy," Journal of Molecular Structure, vol. 883-884, no. 1-3, pp. 155-159, 2008.

[18] H. S. Shin, J. M. Monsallier, and G. R. Choppin, "Spectroscopic and chemical characterizations of molecular size fractionated humic acid," Talanta, vol. 50, no. 3, pp. 641-647, 1999.

[19] I. Christl, C. J. Milne, D. G. Kinniburgh, and R. Kretzschmar, "Relating ion binding by fulvic and humic acids to chemical composition and molecular size. 2. Metal binding," Environmental Science and Technology, vol. 35, no. 12, pp. 2512-2517, 2001.

[20] P. Conte, R. Spaccini, D. Šmejkalová, A. Nebbioso, and A. Piccolo, "Spectroscopic and conformational properties of size-fractions separated from a lignite humic acid," Chemosphere, vol. 69, no. 7, pp. 1032-1039, 2007.

[21] J. Hur, D.-H. Lee, and H.-S. Shin, "Comparison of the structural, spectroscopic and phenanthrene binding characteristics of humic acids from soils and lake sediments," Organic Geochemistry, vol. 40, no. 10, pp. 1091-1099, 2009.

[22] J. Hur and M. A. Schlautman, "Using selected operational descriptors to examine the heterogeneity within a bulk humic substance," Environmental Science and Technology, vol. 37, no. 5, pp. 880-887, 2003.

[23] H. J. Cha, M.-J. Kang, G. H. Chung, G. S. Choi, and C. W. Lee, "Accumulation of 137Cs in soils on different bedrock geology and textures," Journal of Radioanalytical and Nuclear Chemistry, vol. 267, no. 2, pp. 349-355, 2006.

[24] J. Hur and G. Kim, "Comparison of the heterogeneity within bulk sediment humic substances from a stream and reservoir via selected operational descriptors," Chemosphere, vol. 75, no. 4, pp. 483-490, 2009.

[25] I. Noda, A. E. Dowrey, C. Marcott, G. M. Story, and Y. Ozaki, "Generalized two-dimensional correlation spectroscopy," Applied Spectroscopy, vol. 54, no. 7, pp. 236A-248A, 2000.

[26] I. Noda and Y. Ozaki, Two-Dimensional Correlation Spectroscopy, John Wiley \& Sons, West Sussex, UK, 2004.

[27] L. Cavani, S. Halladja, A. T. Halle et al., "Relationship between photosensitizing and emission properties of peat humic acid fractions obtained by tangential ultrafiltration," Environmental Science and Technology, vol. 43, no. 12, pp. 4348-4354, 2009.

[28] C. Richard, O. Trubetskaya, O. Trubetskoj et al., "Key role of the low molecular size fraction of soil humic acids for fluorescence and photoinductive activity," Environmental Science and Technology, vol. 38, no. 7, pp. 2052-2057, 2004.

[29] J. J. Alberts and M. Takács, "Comparison of the natural fluorescence distribution among size fractions of terrestrial fulvic and humic acids and aquatic natural organic matter," Organic Geochemistry, vol. 35, no. 10, pp. 1141-1149, 2004.

[30] J. C. G. Esteves da Silva, A. A. S. C. MacHado, and C. J. S. Oliveira, "Fluorescence quenching of anthropogenic fulvic acids by $\mathrm{Cu}(\mathrm{II}), \mathrm{Fe}(\mathrm{III})$ and $\mathrm{UO}_{2}^{2+}$," Talanta, vol. 45, no. 6, pp. 1155-1165, 1998.

[31] C. Plaza, V. D’Orazio, and N. Senesi, "Copper(II) complexation of humic acids from the first generation of EUROSOILS by total luminescence spectroscopy," Geoderma, vol. 125, no. 1-2, pp. 177-186, 2005.

[32] F. C. Wu, R. D. Evans, and P. J. Dillon, "Fractionation and characterization of fulvic acid by immobilized metal ion affinity chromatography," Analytica Chimica Acta, vol. 452, no. 1, pp. 85-93, 2002.

[33] M. Fukushima, S. Tanaka, H. Nakamura, S. Ito, K. Haraguchi, and T. Ogata, "Copper(II) binding abilities of molecular weight fractionated humic acids and their mixtures," Analytica Chimica Acta, vol. 322, no. 3, pp. 173-185, 1996. 
This article should be cited as follows:

Jin Hur and Bo-Mi Lee, "Comparing the Heterogeneity of Copper-Binding Characteristics for Two Different-Sized Soil Humic Acid Fractions Using Fluorescence Quenching Combined with 2D-COS," TheScientificWorldJOURNAL, vol. 11, pp. 1865-1876, 2011. 


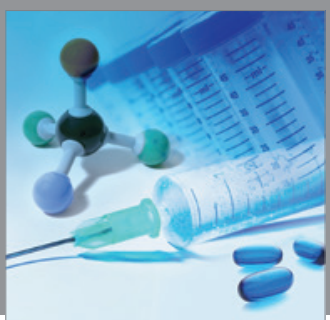

International Journal of

Medicinal Chemistry

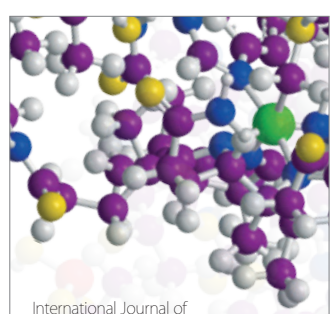

Carbohydrate Chemistry



The Scientific World Journal

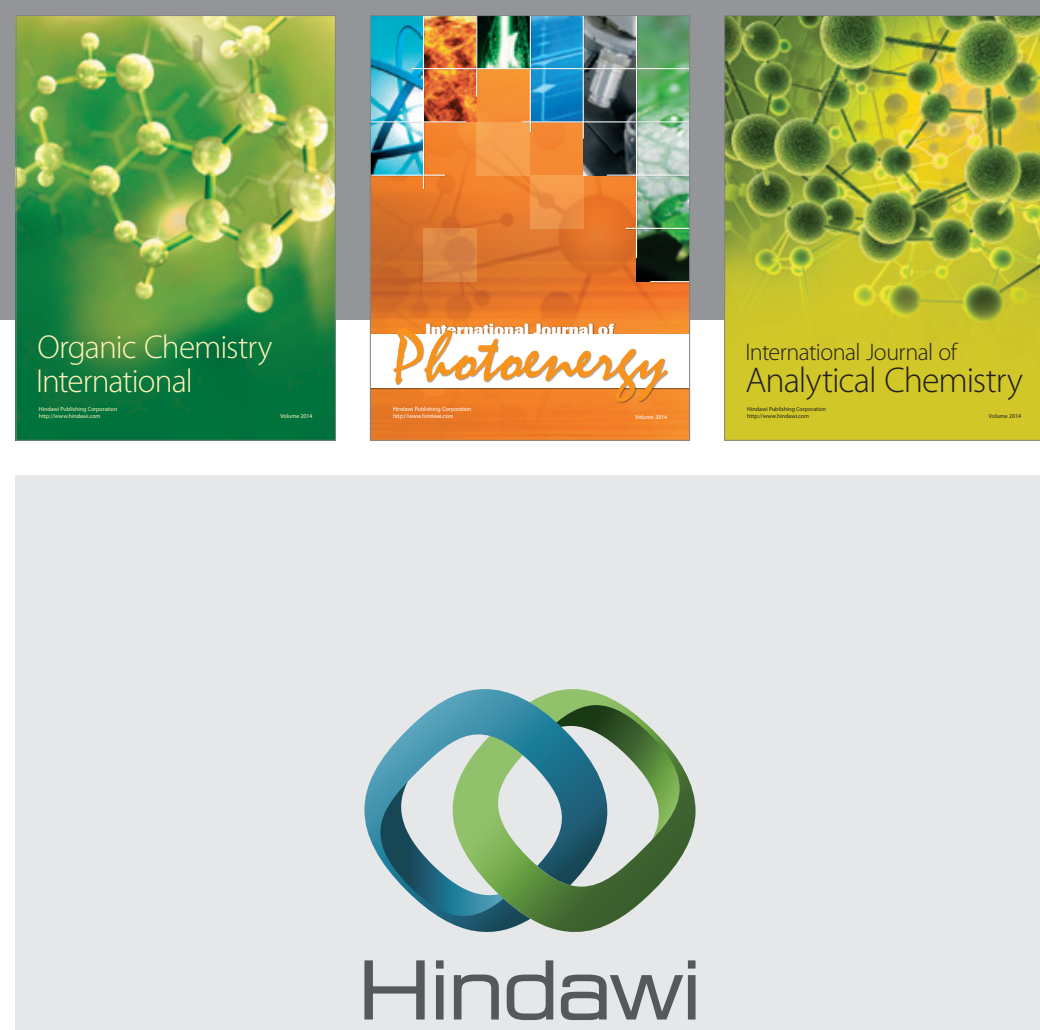

Submit your manuscripts at

http://www.hindawi.com


Journal of

Applied Chemistry
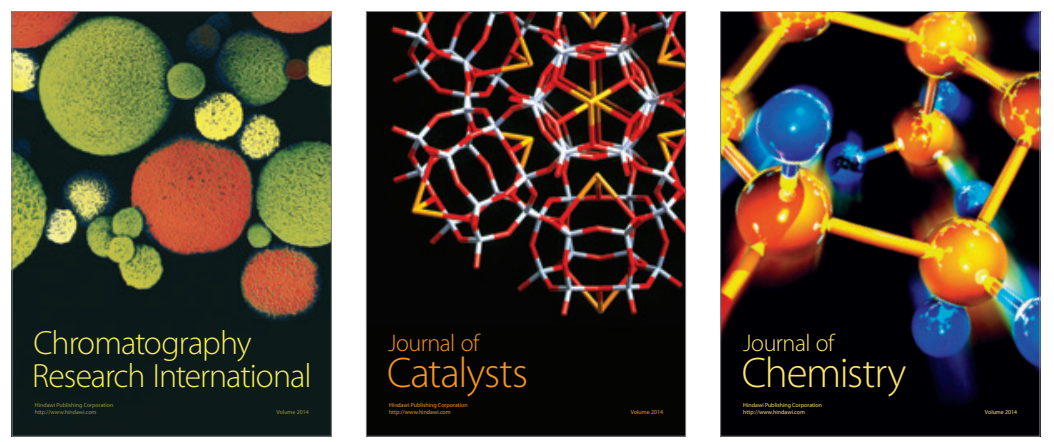
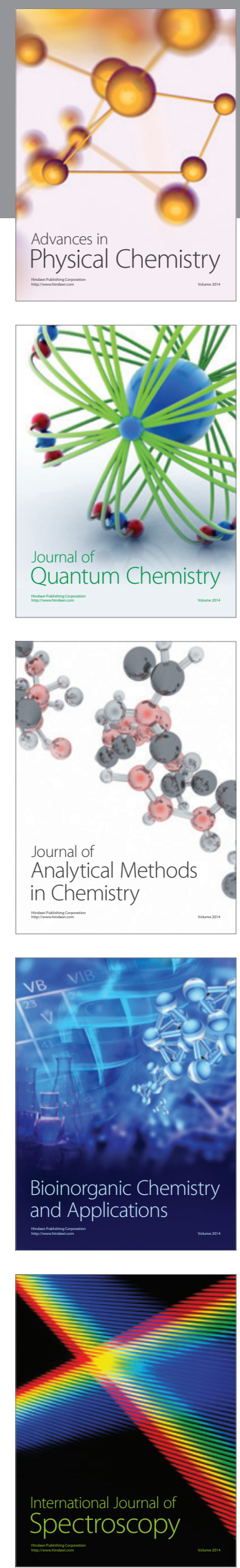